\title{
Nuclear structure functions at low- $x$ in a holographic approach
}

\author{
Luca Agozzino $^{1,2, a}$, Paolo Castorina ${ }^{1,2,3, b}$, Pietro Colangelo ${ }^{4, c}$ \\ ${ }^{1}$ Dipartimento di Fisica, Universitá di Catania, via S. Sofia 62, 95125 Catania, Italy \\ 2 INFN, Sezione di Catania, via S. Sofia 62, 95125 Catania, Italy \\ ${ }^{3}$ PH Department, TH Unit, CERN, 1211 Geneva 23, Switzerland \\ ${ }^{4}$ INFN, Sezione di Bari, via Orabona 4, 70126 Bari, Italy
}

Received: 10 January 2014 / Accepted: 18 March 2014 / Published online: 1 April 2014

(C) The Author(s) 2014. This article is published with open access at Springerlink.com

\begin{abstract}
Nuclear effects in deep inelastic scattering at low $x$ are phenomenologically described changing the typical dynamical and/or kinematical scales characterizing the free nucleon case. In a holographic approach, this rescaling is an analytical property of the computed structure function $F_{2}\left(x, Q^{2}\right)$. This function is given by the sum of a conformal term and of a contribution due to quark confinement, depending on IR hard-wall parameter $z_{0}$ and on the mean square distances, related to a parameter $Q^{\prime}$, among quarks and gluons in the target. The holographic structure function per nucleon in a nucleus $A$ is evaluated showing that a rescaling of the typical nucleon size, $z_{0}$ and $Q^{\prime}$, due to nuclear binding, can be reabsorbed in a $Q^{2}$-rescaling scheme. The difference between neutron and proton structure functions and the effects of the longitudinal structure functions can also be taken into account. The obtained theoretical results favorably compare with the experimental data.
\end{abstract}

\section{Introduction}

Deep inelastic scattering (DIS) experiments of charged leptons off nuclei have shown that the structure functions of nucleons bound in nuclei differ from the structure functions of free, isolated nucleons. Although in some cases a deviation could be expected considering, e.g., the Fermi motion of nucleons in nuclei, in general the interpretation and the predictions of the nuclear modifications have presented considerable difficulties and, not surprisingly, the measurements have generated an intense theoretical and phenomenological activity (for a review see [1,2]).

Nuclear effects can be described comparing the structure functions of the nuclear target, normalized to the number

\footnotetext{
a e-mail: luca.agozzino@ct.infn.it

b e-mail: paolo.castorina@ct.infn.it

c e-mail: pietro.colangelo@ba.infn.it
}

of nucleons, to the free nucleon ones. For electroproduction, if $F_{2}^{D}$ is the structure function of the deuterium $D$ and $F_{2}^{A}$ the structure function per nucleon of the nucleus $A$, the ratio $R_{A}=F_{2}^{A}\left(x, Q^{2}\right) / F_{2}^{D}\left(x, Q^{2}\right)$ is measured for various values of the Bjorken variable $x$ and the squared momentum transferred $Q^{2}$. Nuclear modifications are observed to depend on $x$. For $x \leq 0.1$ the ratio $R_{A}$ is found $R_{A}<1$ : this is the so-called shadowing region. In the range $0.1<x<0.25$ there is antishadowing, with $R_{A}>1$. For large $x$ the so-called EMC effect appears: again a decreasing behavior.

There are different approaches aimed at interpreting such observations. A few of them make use, both for the EMC and the shadowing effect, of the idea that the nuclear modifications are mainly due to the change of the effective mean square distances among quarks and gluons in a nuclear environment with respect to free nucleons (for a review see [1]). Such a geometric modification can be accounted for by a rescaling of the kinematical variables, $x$ or $Q^{2}$, in the structure functions of a free nucleon. This is the case, for example, of the so-called $x$-rescaling model, where the EMC effect is described by rescaling the Bjorken $x$ variable in the free nucleon $F_{2}^{D}[3,4]$ :

$F_{2}^{A}\left(x, Q^{2}\right)=F_{2}^{D}\left(x / \hat{z}, Q^{2}\right)$.

The factor $\hat{z}$ is defined as $\hat{z} \simeq 1-\epsilon / M$, in terms of the proton mass $M$ and of the energy $\epsilon$ necessary to emit a nucleon from a nucleus. A difficulty of this model is that the values of the energy $\epsilon$ to fit the large- $x$ data exceed the calculations of the nuclear binding (for a review see [1]).

The $Q^{2}$-rescaling model of the EMC effect is based on the relation [5-8]

$F_{2}^{A}\left(x, Q^{2}\right)=F_{2}^{D}\left(x, \chi_{A} Q^{2}\right)$,

indicating that the effective $Q^{2}$ for a bound nucleon is different from the free one. Such a dynamical property is related to the modification of the quark confinement scale in the 
nucleus [5-8]: quarks and gluons are no longer confined to specific nucleons, but spread over distances larger than the free nucleon size. By studying the moments of the structure function, starting from a $Q^{2}$ region where the valence picture is a good approximation, one can show that in QCD, for large $Q^{2}$, the change of scale is related to the strong coupling constant $\alpha_{s}$. It is worth remarking that the $x$ - and $Q^{2}$ rescaling models, although different in their assumptions, can be related $[9,10]$.

A different nonperturbative approach considers that the low- $x$ region is governed by the Pomeron exchange $[11,12]$. In a nuclear environment, the nucleon overlap produces a suppression of the effective quark-Pomeron coupling. Indeed, although quarks and gluons are no longer confined to specific nucleons and spread over distances larger than the free nucleon size, the average spatial separation between the quarks before color neutralization decreases, and this reduces the Pomeron coupling which is related to such a typical size [13].

The idea that the description of the nuclear modifications requires to evaluate the change of the free nucleon wave function induced by the nuclear binding can find a support in an analysis based on the holographic approach. The AdS/CFT, or gauge/gravity correspondence principle (see, e.g. [14]), [15-17] is important to access the nonperturbative sector of gauge theories, and can be used to study features of QCD [18-21]. The method has been applied to DIS at strong coupling [22-38]. In particular, at low $x$ the nucleon structure function $F_{2}^{N}\left(x, Q^{2}\right)$ has been computed in Ref. [39], and has been represented as a conformal contribution and an additional term accounting for quark confinement. Both contributions involve the holographic nucleon wave function: since the confinement dynamics determines the modification of the structure functions of a nucleon in nuclei, the holographic baryon wave function in nuclei affects the nuclear structure functions. Following this viewpoint, in the study [40] we attempted a description of shadowing in a gauge/gravity framework, using in the low- $x$ region the AdS/CFT strong coupling BPST Pomeron kernel computed in [41]. The holographic free nucleon wave function is assumed to be peaked at a distance $1 / Q^{\prime}$ close to the boundary $z_{0}$. In the description of the nuclear binding effects, the wave function of the bound nucleon must involve a different effective distance $1 / Q_{A}^{\prime}$ and a new confinement boundary $z_{0}^{A}$. Studying the scaling properties of the holographic expression for $F_{2}$ under the replacement $Q^{\prime} \rightarrow Q_{A}^{\prime}$ and $z_{0} \rightarrow z_{0}^{A}$, nuclear effects turn out to be described by a rescaling of the confinement parameters, with remarkable agreement with measurements.

Here, we discuss this idea in more detail, including the difference between proton and neutron structure functions, analyzing a few approximations adopted in Ref. [40], considering the $x$-rescaling scheme, carrying out a more complete comparison with the experimental data, evaluating the effects of the longitudinal structure function. The paper is organized as follows: in Sect. 2 we review the low- $x$ behavior of the proton structure functions in a holographic approach, and discuss the neutron-proton difference. Section 3 contains the model for the nuclear modifications of the structure functions, which is compared with data in Sect. 3.2. In Sect. 4 we discuss the longitudinal structure function in nuclei, and in Sect. 5 we present our conclusions.

\section{Holographic proton structure functions}

The AdS/CFT calculation of DIS at low $x$ on a proton was first considered by Polchinski and Strassler in [22,23]. After this seminal proposal, several calculations have been carried out in various holographic frameworks [22-38]. In particular, in [39] the nucleon structure function $F_{2}$ was computed analyzing the virtual $\gamma^{*} p$ total cross section, and two contributions were obtained, a term for conformal gauge theories and an additional term accounting for confinement. A slice of the dual AdS space was used to break the conformal invariance. As shown in [40], this result can be used to analyze nuclear effects on $F_{2}$.

The definition in QCD of the structure functions $F_{1}\left(x, Q^{2}\right)$ and $F_{2}\left(x, Q^{2}\right)$ of a hadron of momentum $P$ and charge $\mathscr{Q}$ is based on the matrix element of two electromagnetic currents

$T^{\mu \nu} \equiv i \int d^{4} y e^{i q \cdot y}\left\langle P \mathscr{Q}\left|T\left(J^{\mu}(y) J^{\nu}(0)\right)\right| P \mathscr{Q}\right\rangle$,

which can be written as

$$
\begin{aligned}
T^{\mu \nu}= & F_{1}\left(x, Q^{2}\right)\left(\eta^{\mu \nu}-\frac{q^{\mu} q^{\nu}}{q^{2}}\right) \\
& +\frac{2 x}{q^{2}} F_{2}\left(x, Q^{2}\right)\left(P^{\mu}+\frac{q^{\mu}}{2 x}\right)\left(P^{\nu}+\frac{q^{\nu}}{2 x}\right) .
\end{aligned}
$$

$\mu, v$ are four-dimensional indices, $\eta^{\mu \nu}$ the Minkowski metric, the Bjorken variable $x$ is $x=\frac{Q^{2}}{2 P \cdot q}$, with $Q^{2}=-q^{2}$.

The AdS/CFT calculation involves $R$-currents in (3), and the couplings

$g_{s}=\frac{g_{Y M}^{2}}{4 \pi}=\alpha_{Y M}=\frac{\lambda}{4 \pi N_{C}}, \quad R=\alpha^{\prime \frac{1}{2}} \lambda^{\frac{1}{4}}$.

$g_{Y M}$ is the Yang-Mills coupling constant, $N_{C}$ the number of colors, in the regime $g_{s}<<1$ and $\lambda>>1 . R$ is the AdS radius.

The dual string calculation of the matrix element (3), or of its imaginary part appearing in DIS processes, describes the photon-hadron scattering $\gamma^{*} p \rightarrow \gamma^{*} p \equiv 1,2 \rightarrow 3,4$ as occurring in the AdS space. Various quantities are needed, starting from the states dual to the initial-final hadron $p$. For protons, these states are represented by normalizable wave functions $\phi^{p}(z)$, in principle obtained from a suitable equa- 
tion of motion, with some dependence on the holographic coordinate $z$. For the calculation of the matrix element (3) the transition function is required:

$P_{24}(z)=\sqrt{-g}\left(\frac{z}{R}\right)^{2} \phi^{p}(z) \phi^{p}(z)$.

The current that couples to the hadrons in the matrix element (3) excites non-normalizable modes of the gauge fields $\mathscr{A}$, which in the bulk obey Maxwell's equations. In the Lorentz gauge and for $R=1$ there are the solutions: $\mathscr{A}_{\mu}(y, z)=n_{\mu}(Q z) K_{1}(Q z) e^{i q \cdot y}$ and $\mathscr{A}_{z}(y, z)=i(q$. $n)(Q z) K_{0}(Q z) e^{i q \cdot y}$, given in terms of Bessel functions $K_{1}$ and $K_{0}$ and of the polarization vector $n_{\mu}$. The calculation of the structure function $F_{2}$ in $(3,4)$ requires the transition function

$P_{13}\left(z, Q^{2}\right)=\frac{1}{z}(Q z)^{2}\left[K_{0}^{2}(Q z)+K_{1}^{2}(Q z)\right]$,

with $Q=\sqrt{Q^{2}}$, while

$P_{13}\left(z, Q^{2}\right)=\frac{1}{z}(Q z)^{2} K_{1}^{2}(Q z)$

is needed for $2 x F_{1}$. From now on, we focus on $F_{2}$ : the effect of the nuclear modification on the longitudinal structure function $F_{L}=F_{2}-2 x F_{1}$ will be discussed in Sect. 4 .

Finally, the scattering kernel is needed. Expressing it in terms of a Pomeron Regge pole contribution [41], at low $x$ the structure function $F_{2}$ can be written as an eikonal sum [39]:

$$
\begin{aligned}
F_{2}^{p}\left(x, Q^{2}\right)= & \frac{Q^{2}}{2 \pi^{2}} \int d^{2} b \int d z d z^{\prime} P_{13}\left(z, Q^{2}\right) P_{24}\left(z^{\prime}\right) \\
& \times \operatorname{Re}\left(1-e^{i \chi\left(s, b, z, z^{\prime}\right)}\right) .
\end{aligned}
$$

$s$ is the center-of-mass energy squared of the $\gamma^{*}$-target system and $b$ the impact parameter. The derivation of the eikonal $\chi$ for conformal theories and including conformal breaking effects is in Refs. [39,41].

\subsection{Conformal term}

An expression of the proton structure function $F_{2}^{p}$ in the conformal case, derived from Eq. (9), as been worked out in Ref. [39]:

$$
\begin{aligned}
F_{2 c f}^{p}\left(x, Q^{2}\right)= & \frac{g_{0}^{2} \rho^{3 / 2}}{32 \pi^{5 / 2}} \int d z d z^{\prime} \frac{z z^{\prime} Q^{2}}{\tau^{1 / 2}} P_{13}\left(z, Q^{2}\right) P_{24}\left(z^{\prime}\right) \\
& \times e^{(1-\rho) \tau} \exp \left[\Phi\left(z, z^{\prime}, \tau\right)\right] .
\end{aligned}
$$

$g_{0}^{2}$ is a parameter and $x \simeq Q^{2} / s ; \rho$ is defined in terms of the 't Hooft coupling in (5), $\rho=2 / \sqrt{\lambda}$. The function $\tau$, defined as $\tau=\log \left(\rho z z^{\prime} s / 2\right)$, is a conformal invariant. $\Phi$ is the BPTS Pomeron kernel integrated in impact parameter [41]:
$\Phi\left(z, z^{\prime}, \tau\right)=-\frac{\left(\log z-\log z^{\prime}\right)^{2}}{\rho \tau}$.

Equations (9) and (10) involve the transition functions $P_{24}$ and $P_{13}$. The proton wave function in the bulk $\phi^{p}(z)$, needed in $P_{24}$, should be determined by an explicit holographic model for the baryon. An approximation has been used in Ref. [39], assuming that $\phi^{p}(z)$ is peaked close to the infrared boundary $z_{0}$, with $1 / Q^{\prime} \leq z_{0}$ and $Q^{\prime}$ of the order of nucleon mass, giving

$P_{24}\left(z^{\prime}\right) \simeq \delta\left(z^{\prime}-\frac{1}{Q^{\prime}}\right)$.

Moreover, also $P_{13}$ can be replaced by a local expression

$P_{13}\left(z, Q^{2}\right) \simeq C \delta\left(z-\frac{1}{Q}\right)$,

with $C \simeq 1$ [39]. This is justified by the shape of the function $P_{13}$ in Eq. (10), which is peaked for $z \simeq 1 / Q$. In Fig. 1 we depict $F_{2 c f}^{p}$ obtained using the exact expression in Eq. (7) and the local approximation Eq. (13) for two values of the squared transferred momentum: the relative difference between the two expressions is within a few percent for $x<0.07$.

The resulting $F_{2 c f}^{p}$ reads [39]

$F_{2 c f}^{p}\left(x, Q^{2}, Q^{\prime}\right)=\frac{g_{0}^{2} \rho^{3 / 2}}{32 \pi^{5 / 2}} \frac{Q}{Q^{\prime}} \frac{e^{(1-\rho) \tau}}{\tau^{1 / 2}} e^{-\left[\log ^{2}\left(Q / Q^{\prime}\right) / \rho \tau\right]}$.

\subsection{Confinement term}

The expression for the proton structure function $F_{2 c f}^{p}$, based on the conformal BPST Pomeron, does not fit the HERA data in the low- $Q^{2}$ range, where confinement is the main dynamical mechanisms [39]. Confinement can be described in the holographic approach including an infrared boundary $z_{0}$ on the $z$ bulk coordinate, a so-called hard-wall holographic

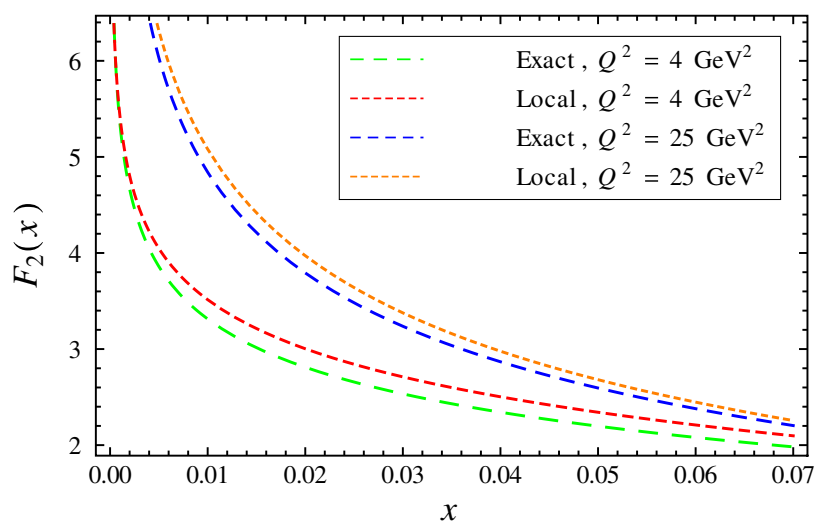

Fig. 1 Comparison between $F_{2 c f}^{p}$ in Eq. (10), obtained using Eq. (7) (labeled as Exact) and the approximation Eq. (13) (labeled as Local), for $Q^{2}=4$ and $25 \mathrm{GeV}^{2}$ 
model of QCD. This confinement scale can be related to $\Lambda_{\mathrm{QCD}}$. The eikonal is modified and a non-conformal contribution to $F_{2}^{p}$ should be considered, which reads for a single Pomeron [39]

$$
\begin{aligned}
F_{2 c t}^{p}\left(x, Q^{2}, z_{0}\right)= & \frac{g_{0}^{2} \rho^{3 / 2}}{32 \pi^{5 / 2}} \int d z d z^{\prime} \frac{z z^{\prime} Q^{2}}{\tau^{1 / 2}} P_{13}\left(z, Q^{2}\right) P_{24}\left(z^{\prime}\right) \\
& \times e^{(1-\rho) \tau} e^{-\frac{\log ^{2}\left(z z^{\prime} / z_{0}^{2}\right)}{\rho \tau}} G\left(z, z^{\prime}, \tau\right) .
\end{aligned}
$$

The $z_{0}$ dependence is shown explicitly. The function $G\left(z, z^{\prime}, \tau\right)$ is

$G\left(z, z^{\prime}, \tau\right)=1-2 \sqrt{\rho \pi \tau} e^{\eta^{2}} \operatorname{erfc}(\eta)$,

with

$\eta=\frac{-\log \left(z z^{\prime} / z_{0}^{2}\right)+\rho \tau}{\sqrt{\rho \tau}}$.

Adopting the approximation (12) and (13), Eq. (15) reduces to

$$
\begin{aligned}
F_{2 c t}^{p}\left(x, Q^{2}, Q^{\prime}, Q_{0}^{2}\right)= & \frac{g_{0}^{2} \rho^{3 / 2}}{32 \pi^{5 / 2}} \frac{Q}{Q^{\prime}} \frac{e^{(1-\rho) \tau}}{\tau^{1 / 2}} \\
& \times e^{-\frac{\log ^{2}\left(Q_{0}^{2} /\left(Q Q^{\prime}\right)\right)}{\rho \tau}} G\left(\frac{1}{Q}, \frac{1}{Q^{\prime}}, \tau\right),
\end{aligned}
$$

with $Q_{0}=1 / z_{0}$ [39].

The proton structure function $F_{2}^{p}$ results from the sum of the conformal and confinement contribution,

$F_{2}^{p}\left(x, Q^{2}\right)=F_{2 c l}^{p}\left(x, Q^{2}, Q^{\prime}\right)+F_{2 c t}^{p}\left(x, Q^{2}, Q^{\prime}, Q_{0}^{2}\right)$,

and can successfully be compared with proton DIS data [39].

It is interesting to analyze the relative weight of the conformal and confinement contributions to $F_{2}^{p}$ at low $x$. In Fig. 2 three values of $Q^{2}$ are considered: at $Q^{2} \simeq 4 \mathrm{GeV}^{2}$ the structure function is essentially determined by the conformal term. On the other hand, the confinement term is the main contribution at very low $Q^{2}$ for all the considered values of the Bjorken $x$.

\subsection{Accounting for isospin effects: neutron structure function}

Isospin effects play an important role in detailed analyses of nuclear structure functions (normalized to the total number of nucleons). These effects represent the difference between the proton and neutron structure function. In the holographic model, the difference can be implemented in a rather simple way replacing the scales $Q_{0}$ and $Q^{\prime}$ for the proton with corresponding scales $Q_{0 n}$ and $Q_{n}^{\prime}$ for the neutron. Therefore, the neutron structure function $F_{2}^{n}$ can be represented by the expression

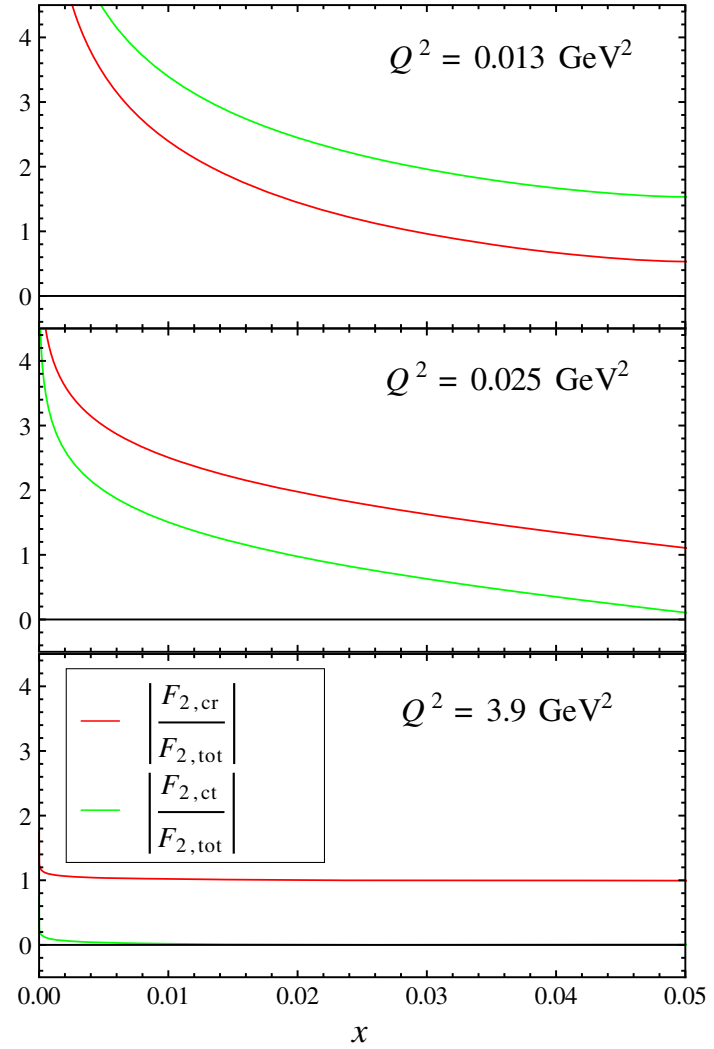

Fig. 2 Comparison between conformal and confinement contributions to $F_{2}^{p}\left(x, Q^{2}\right)$ at low $x$, for three values of $Q^{2}$. The red (dark) lines correspond to the absolute value $\left|F_{2 c f}^{p} / F_{2}^{p}\right|$ of the ratio of the conformal term $F_{2 c f}^{p}$ in Eq. (19) over the full structure function; the green (light) lines correspond to ratio $\left|F_{2 c t}^{p} / F_{2}^{p}\right|$ of the confinement contribution $F_{2 c t}^{p}$ to the full structure function. For the lowest value of $Q^{2}$ the confinement term dominates

$F_{2}^{n}=F_{2 c l}^{p}\left(x, Q^{2}, Q_{n}^{\prime}\right)+F_{2 c t}^{p}\left(x, Q^{2}, Q_{n}^{\prime}, Q_{0 n}^{2}\right)$,

with $Q_{0 n} \simeq Q_{0}$, since the proton and neutron have a similar color confinement scale.

The experimental information on the neutron structure function comes from DIS on a deuterium target; therefore, the comparison of the expression (20) with data requires implementing the nuclear effects discussed in the next section. Here we anticipate the proposal to describe the isospin difference in the holographic formula mainly through the parameter $Q_{n}^{\prime}$.

\section{Nuclear structure functions in holographic framework}

In the Introduction we have mentioned that a physical description of the EMC and of the shadowing effects can be obtained considering an effective modification of the dynamical length/momentum scales in deep inelastic scattering pro- 
cesses on a nuclear target with respect to a free nucleon. It is remarkable that such a rescaling, in particular the $Q^{2}$ rescaling, is a property of the analytic expression of the holographic structure function, not only in the conformal term but also in the term taking the confinement dynamics into account.

Let us focus on the conformal contribution (14) to $F_{2}^{N}$ ( $N=$ nucleon, neglecting for the moment the protonneutron difference), which depends on the ratio $Q / Q^{\prime}$. The description of the modification of the structure function (per nucleon) $F_{2}^{A}$ in the nucleus $A$, using the rescaling

$Q_{A}^{\prime}=\lambda_{A} Q^{\prime}$,

corresponds to the rescaling $Q^{2} \rightarrow Q^{2} / \lambda_{A}^{2}$. In (21) $Q_{A}^{\prime}$ is identified with the typical scale of the wave function of the bound nucleon. Consequently, one has

$F_{2 c f}^{A}\left(x, Q^{2}\right)=F_{2 c f}^{N}\left(x, \frac{Q^{2}}{\lambda_{A}^{2}}, Q^{\prime}\right)$

and the $Q^{2}$-rescaling at low $x$ naturally arises in the conformal contribution to the holographic expression of $F_{2}$.

In the confinement term in Eqs. (15), (16), and (17) a nontrivial $Q^{2}$ behavior appears in the log-factors and in $\eta$, due to the infrared scale $Q_{0}$. The rescaling $Q_{A}^{\prime}=\lambda_{A} Q^{\prime}$ can be reabsorbed in the $Q^{2}$ rescaling, $Q^{2} \rightarrow Q^{2} / \lambda_{A}^{2}$, as in the conformal term. Since the dependence on $Q_{0}$ in Eqs. (15), (16) and (17) is in the combination $Q_{0}^{2} / Q Q^{\prime}$, the modification $Q_{A}^{\prime}=\lambda_{A} Q^{\prime}$ can be reabsorbed in the same $Q^{2}$ rescaling also in the confinement term, provided that the confinement length in the nuclear environment scales in the same way:

$Q_{0}^{2} \rightarrow Q_{0}^{2} / \lambda_{A}^{2}$

The origin of the rescaling (21) and (23) in the AdS/CFT framework comes from the identification of the bulk coordinate with the energy scale of the dual theory: from the form of the $A d S$ metric in Poincaré coordinates, a coordinate rescaling $x_{\mu} \rightarrow \lambda x_{\mu}$ on the boundary corresponds to $z \rightarrow \lambda z$ in the bulk. In nuclei, due to the nucleon overlap, the average distance among quarks and gluons decreases and the color neutralization infrared (confinement) scale increases. These modifications in the boundary correspond in the bulk, respectively, to $z^{\prime} \rightarrow z^{\prime} / \lambda$ and $z_{0} \rightarrow \lambda z_{0}$ : these are the prescription (21) and (23) used to describe the nuclear effects by redefining the momenta.

In our phenomenological analysis, the following expression of the structure function $F_{2}^{A}$ (per nucleon) in the nucleus $A$ will be used:

$F_{2}^{A}\left(x, Q^{2}\right)=F_{2 c l}^{N}\left(x, \frac{Q^{2}}{\lambda_{A}^{2}}, Q^{\prime}\right)+F_{2 c t}^{N}\left(x, \frac{Q^{2}}{\lambda_{A}^{2}}, Q^{\prime}, \frac{Q_{0}^{2}}{\lambda_{A}^{2}}\right)$.

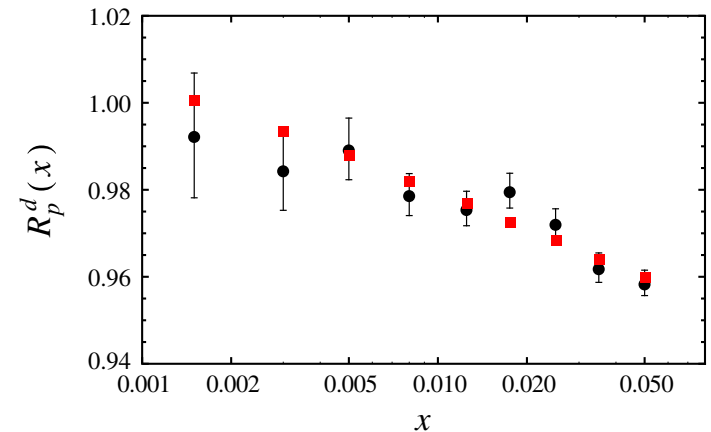

Fig. 3 Comparison between the measurements of the ratio of deuterium and proton structure functions $F_{2}^{D} / F_{2}^{p}$ (black points) [42] and the expression obtained by Eqs. (25), (26), and (27) (red squares). In the theoretical formula, the experimental average $Q^{2}$ for given $x$ is used: the $Q^{2}$ values (in $\mathrm{GeV}^{2}$ ), from the first to the last bin in $x$, vary in the range [0.37-5.8]. The $\chi^{2}$ of the fit is $\chi^{2} /$ d.o.f. $=0.85$

This formula involves the parameter $\lambda_{A}$, specific of the various nuclei, to be fitted from data; moreover, one has to include the proton-neutron difference, discussed below.

\subsection{Deuterium structure function}

Accounting for the isospin effects is required in the analysis of nuclear DIS data. We implement such effects using the neutron $Q_{0 n}$ and $Q_{n}^{\prime}$ scales, and representing the structure function $F_{2}^{D}$ (per nucleon) in deuterium as

$F_{2}^{D}=\frac{1}{2}\left[F_{2}^{p D}+F_{2}^{n D}\right]$

where

$$
\begin{aligned}
& F_{2}^{p D}=F_{2}^{p}\left(x, \frac{Q^{2}}{\lambda_{D}^{2}}, Q^{\prime}, \frac{Q_{0}}{\lambda_{D}^{2}}\right), \\
& F_{2}^{n D}=F_{2}^{n}\left(x, \frac{Q^{2}}{\lambda_{D}^{2}}, Q_{n}^{\prime}, \frac{Q_{0 n}}{\lambda_{D}^{2}}\right) .
\end{aligned}
$$

Since deuterium is a weakly bound system, nuclear effects are small, and one expects $\lambda_{D} \simeq 1$. Indeed, a best fit to data of the expression (25), shown in Fig. 3, is obtained for $\lambda_{D}=1.011$, with $Q_{0 n}=0.192713$ and $Q_{n}^{\prime}=0.177866$. Using these values of $Q_{n}^{\prime}$ and $Q_{0 n}$ together with the corresponding parameters for the proton: $Q_{0 p}=0.201613$ and $Q_{p}^{\prime}=0.4333$ [39], the neutron/proton ratio is determined and can be favorably compared to data in Figs. 3 and 4. As expected, the proton and neutron confinement scales $Q_{0}$ nearly coincide.

\subsection{Heavy nuclei}

Before analyzing the nuclear DIS data, it is worth pointing out that nuclear modifications of the structure functions for heavy nuclei have important phenomenological consequences. A 


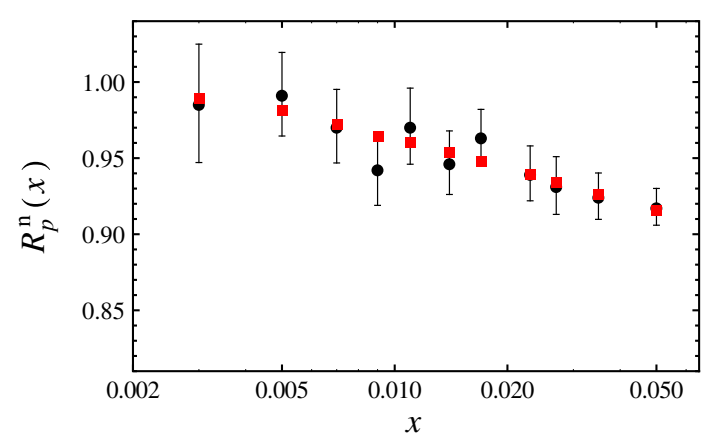

Fig. 4 Experimental measurements of the ratio $F_{2}^{n} / F_{2}^{p}$ (black points) [43] compared to the ratio of the neutron and proton structure function evaluated by Eq. (20) (red squares). In the theoretical expression, the experimental average $Q^{2}$ for given $x$ is used: from the first to the last bin in $x$, the experimental average $Q^{2}$ (in $\mathrm{GeV}^{2}$ ) varies in the range $[0.4-2.6]$. The $\chi^{2}$ of the fit is $\chi^{2} /$ d.o.f. $=0.23$

prime example is the identification of the experimental signatures of the formation of a possible new state of matter in relativistic heavy ion collisions, at the CERN Large Hadron Collider (LHC) and at the Brookhaven RHIC. The identification requires a detailed control of the background processes. In the investigation of a possible new state of matter, the socalled "hard-probes" are crucial, i.e. the dynamical processes originating from hard-parton scattering. The experimental analyses are focused on the differences in the same phenomenon (jet production, $J / \psi$ suppression, etc.) observed in nucleus-nucleus collisions with respect to proton-proton and proton-nucleus scattering, where the obtained energy density is not enough to produce the transition to the new phase. Since the hard-parton scattering involves the parton distribution functions (pdfs), statements on the experimental signature of the new state of matter using hard-probes crucially depend on the control on the modifications of structure functions induced by the ordinary nuclear dynamics [44-46].

Coming to the analysis of nuclear DIS data, the holographic expression of $F_{2}^{A}$ for a nucleus with charge $Z$ can be written as

$$
\begin{aligned}
F_{2}^{A}\left(x, Q^{2}\right)= & \left(\frac{Z}{A}\right) F_{2}^{p}\left(x, \frac{Q^{2}}{\lambda_{A}^{2}}, Q^{\prime}, \frac{Q_{0}}{\lambda_{A}^{2}}\right) \\
& +\left(1-\frac{Z}{A}\right) F_{2}^{n}\left(x, \frac{Q^{2}}{\lambda_{A}^{2}}, Q_{n}^{\prime}, \frac{Q_{0}}{\lambda_{A}^{2}}\right),
\end{aligned}
$$

with the proton and neutron structure functions in Eqs. (19) and (20), and the scaling parameter $\lambda_{A}$ accounting for the nuclear modification. For different nuclei, the ratio $R_{A}=$ $F_{2}^{A} / F_{2}^{D}$ can be analytically evaluated at small $Q^{2}$ and small $x$, in a regime where the perturbative approach cannot be applied. The results can be compared to the experimental data, using the data sets in Table 1 for the various nuclei, together with the values of $\lambda_{A}$ in Table 2. The comparison is shown in Figs. 5 and 6. Considering the $\chi^{2} /$ d.o.f. reported in
Table 1 Experimental data sets [47-49] and $\chi_{\text {d.o.f. }}^{2}$ of the fit of the structure function $F_{2}^{A}$ for each nucleus. The third column reports the $\chi_{\text {d.o.f. }}^{2}$ of fits without isospin breaking, the fourth and fifth columns correspond to fits with the isospin-breaking effect included. In the last column, the experimental average $Q^{2}$ ranges (in $\mathrm{GeV}^{2}$ ) for the various cases are indicated, from the first to the last bin of the Bjorken $x$

\begin{tabular}{lllcll}
\hline Nucleus & no. points & $\chi_{\text {d.o.f. }}^{2}$ & n. points & $\chi_{\text {d.o.f. }}^{2}$ & Range of $\left\langle Q^{2}\right\rangle$ \\
\hline $\mathrm{He}$ & 9 & 1.09 & 9 & 0.24 & {$[0.77-6.3]$} \\
$\mathrm{Li}$ & 9 & 0.93 & 9 & 0.79 & {$[0.03-1.4]$} \\
$\mathrm{Be}$ & 6 & 0.21 & 6 & 0.30 & {$[3.4-11.4]$} \\
$\mathrm{C}$ & 9 & 1.61 & 15 & 0.89 & {$[0.03-6.4]$} \\
$\mathrm{Al}$ & 6 & 0.23 & 6 & 0.21 & {$[3.4-11.6]$} \\
$\mathrm{Ca}$ & 9 & 8.0 & 9 & 3.87 & {$[0.6-6.8]$} \\
$\mathrm{Fe}$ & 6 & 0.41 & 6 & 0.42 & {$[3.4-11.8]$} \\
$\mathrm{Pb}$ & 6 & 1.11 & 6 & 0.93 & {$[3.4-11.6]$} \\
\hline
\end{tabular}

Table 2 Rescaling parameter $\lambda_{A}$ obtained using the holographic expression for $F_{2}^{A}$ and taking into account the isospin breaking. The values in the last column are obtained within the QCD dipole model $[52,53]$

\begin{tabular}{lll}
\hline Nucleus & $\lambda_{A}$ (holography) & $\lambda_{A, \text { dip }}[52,53]$ \\
\hline $\mathrm{Li}$ & 1.843 & 1.130 \\
$\mathrm{Be}$ & 1.764 & 1.140 \\
$\mathrm{C}$ & 1.775 & 1.160 \\
$\mathrm{Al}$ & 1.972 & 1.264 \\
$\mathrm{Ca}$ & 2.006 & 1.338 \\
$\mathrm{Fe}$ & 2.090 & 1.413 \\
$\mathrm{~Pb}$ & 2.286 & 1.780 \\
\hline
\end{tabular}

Table 1 for each nucleus, the agreement of the theoretical formula with data is remarkable, and the $x$-dependence exhibited by data is closely followed by the theoretical results.

It is interesting to comment on the isospin-breaking effects, since fits of the nuclear structure functions could also be done neglecting the proton-neutron difference. The inclusion of the isospin effect improves the accuracy of the fits, as one can infer from the various $\chi_{\text {d.o.f. }}^{2}$ in Table 1; the only exceptions are $\mathrm{Be}$ and $\mathrm{Fe}$, where $\chi_{\text {d.o.f. }}^{2}$ remain essentially unchanged if the isospin breaking is considered.

\section{$3.3 x$-rescaling}

We have shown that, in the holographic approach, the nucleon structure function $F_{2}^{A}$ at low $x$ in a nuclear environment can be obtained rescaling the effective lengths appearing in the nucleon wave function in nuclei. It is interesting to notice that, using the local approximation (12) and (13), one has the combination $\tau=\log \left(\rho Q / 2 x Q^{\prime}\right)$. Therefore, the rescaling $Q_{A}^{\prime}=\lambda_{A} Q^{\prime}$ could be reabsorbed not in the $Q^{2}$ rescaling, but rather in $x \rightarrow \lambda_{A} x$. However, due to the $Q^{2}$ dependence 

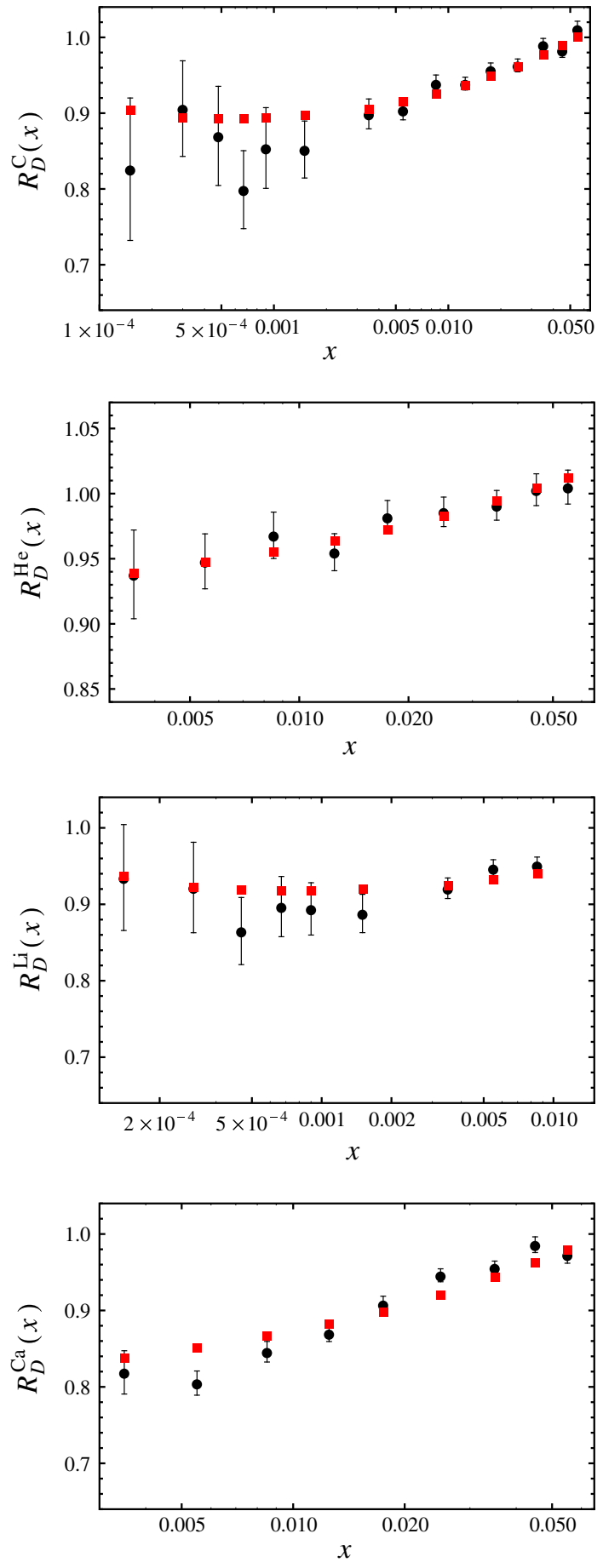

Fig. 5 Ratio $F_{2}^{A} / F_{2}^{D}$ for various nuclei. The black points correspond to the experimental measurements with the data sets in Table 1, the red boxes to the holographic formulas with parameters $\lambda_{A}$ in Table 2 . The isospin-breaking effect has been taken into account. From top-down, the panels correspond to: $\mathrm{C} / \mathrm{D}, \mathrm{He} / \mathrm{D}, \mathrm{Li} / \mathrm{D}, \mathrm{Ca} / \mathrm{D}$. The $\chi_{\text {d.o.f. }}^{2}$ of the fit of the structure functions is in Table 1

of $F_{2}$ in Eq. (14), the $x$-rescaling is not equivalent to the $Q^{2}$ rescaling, and consequently $F_{2}^{A}\left(x, Q^{2} / \lambda^{2}\right) \neq F_{2}^{A}\left(\lambda x, Q^{2}\right)$. One can wonder if the $x$-rescaling is in agreement with the
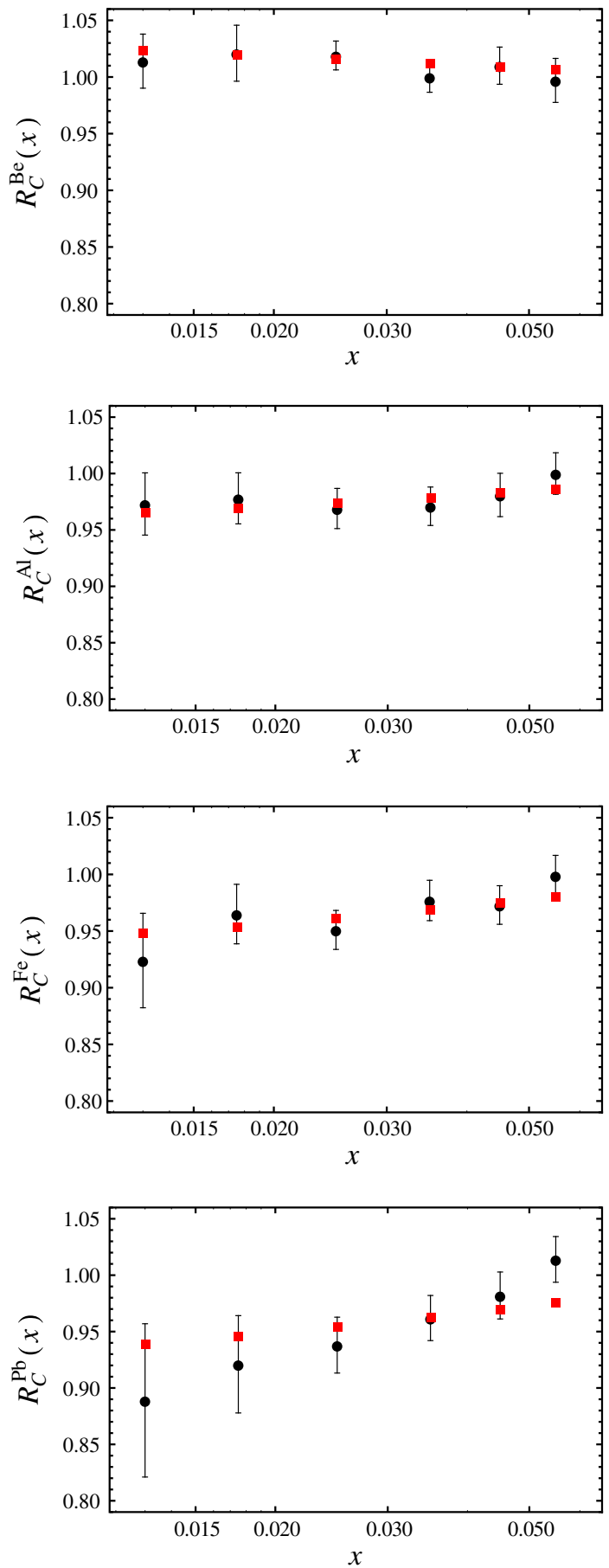

Fig. 6 Ratio $F_{2}^{A} / F_{2}^{C}$ for various nuclei. The black points correspond to the experimental measurements with the data sets in Table 1, the red boxes to the holographic formulas with parameters $\lambda_{A}$ in Table 2 . The isospin-breaking effect has been taken into account. From top-down, the panels correspond to: $\mathrm{Be} / \mathrm{C}, \mathrm{Al} / \mathrm{C}, \mathrm{Fe} / \mathrm{C}, \mathrm{Pb} / \mathrm{C}$. The $\chi_{\text {d.o.f. }}^{2}$ are in Table 1

data: looking at Fig. 7 we conclude that this is not the case, not surprisingly, since the $x$-rescaling has been proposed as a possible explanation of the EMC effect at large- $x$. 


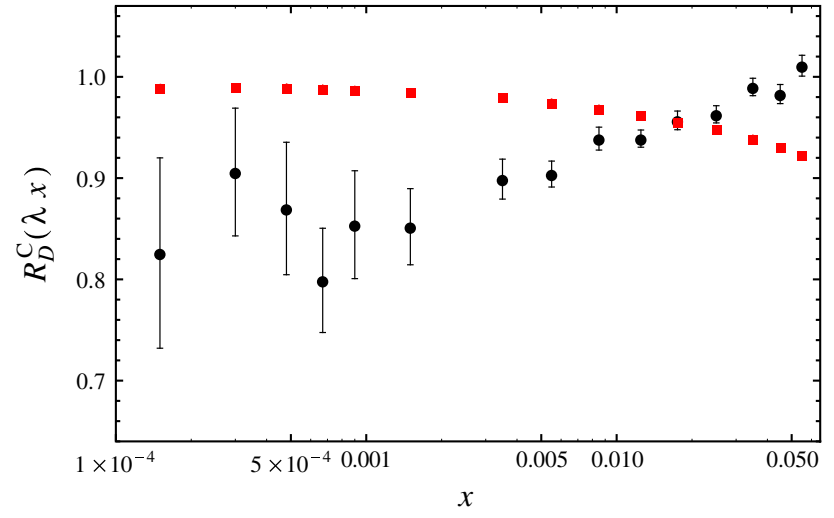

Fig. 7 Comparison of experimental data for the ratio $F_{2}^{C} / F_{2}^{D}$ (black points) with the result obtained by $x$-rescaling in the holographic expression of the structure function (red boxes)

\subsection{Remarks}

As we have discussed, in the holographic formula the nuclear effects in the DIS structure functions can be described by a $Q^{2}$-rescaling, corresponding to a modification of the confinement length for a bound nucleon. Other different methods produce similar results. An example is the QCD dipole model [50,51], where the structure functions are determined considering a virtual photon $\gamma^{*}$ splitting in a quark-antiquark dipole which interacts with the target $T$. Encoding the energy and target size dependence of the dipole-target cross section $\sigma^{\gamma^{*} T}$ in the saturation scale $Q_{S, T}(x)[52,53], \sigma^{\gamma^{*} T}$ turns out to depend only on the ratio $\tau_{T}^{2}=Q^{2} / Q_{S, T}^{2}(x)$. This implies a geometric scaling between the nucleus and the nucleon cross sections [52,53]:

$\frac{\sigma^{\gamma^{*} A}\left(\tau_{A}\right)}{\pi R_{A}^{2}}=\frac{\sigma^{\gamma^{*} N}\left(\tau_{N}\right)}{\pi R_{N}^{2}}$,

with radii $R_{N, A}$ and

$\tau_{A}^{2}=\tau_{N}^{2}\left(\frac{\pi R_{A}^{2}}{A \pi R_{N}^{2}}\right)^{1 / \delta}$.

The consequence is

$Q_{S, A}^{2}=Q_{S, N}^{2}\left(\frac{A \pi R_{N}^{2}}{\pi R_{A}^{2}}\right)^{1 / \delta}$.

Since the cross section only depends on $Q^{2} / Q_{S, T}^{2}(x)$, the replacement $Q_{S, N}^{2} \rightarrow Q_{S, A}^{2}$ corresponds to rescaling

$Q^{2} \rightarrow Q^{2} / \lambda_{A, \text { dip }}^{2}$,

with

$\lambda_{A, d i p}=\left(\frac{A \pi R_{N}^{2}}{\pi R_{A}^{2}}\right)^{1 / 2 \delta}$.
In the dipole model low- $x$ nuclear data are reproduced for $R_{A}=\left(1.12 A^{1 / 3}-0.86 A^{-1 / 3}\right) \mathrm{fm}, \pi R_{N}^{2}=1.55 \mathrm{fm}^{2}$, and $\delta=0.79[52,53]$.

In Table 2 we compare the rescaling parameters $\lambda_{A}$ obtained in the holographic and in the QCD dipole model. Regardless of the difference between the two theoretical approaches, the rescaling parameters differ by less than 30 $35 \%$; however, the deviation is larger than in the case where the isospin breaking is neglected [40].

It would be interesting to extend the analysis to the antishadowing region, for which no dynamical description is available at present, and the comparisons with experimental data are based on the energy-momentum sum rule for the modified parton distribution functions (studies of the energymomentum tensor in the framework of AdS/QCD, with applications to nucleon properties, can be found in [54]). This study is not a straightforward application of the methods described above. Indeed, antishadowing probably requires a different rescaling mechanism, and we defer it to a future dedicated investigation.

\section{Nuclear modification of the longitudinal structure function}

The experimental determination of the structure function per nucleon in a nucleus is usually done by cross section data, assuming a minor nuclear effect on the longitudinal structure function $F_{L}=F_{2}-2 x F_{1}$, hence using the value of the free nucleon $F_{L}^{N}$. This procedure has to be checked, because it introduces an uncertainty in the evaluation of the nuclear structure functions which, in turn, implies an uncertainty in the determination of the modified pdfs.

A holographic expression for the longitudinal structure function $F_{L}$ can obtained from Eqs. (10) and (15), using the local approximation for $P_{24}$, and for $P_{13}$

$\left.P_{13}\left(z, Q^{2}\right)\right|_{F_{L}}=\frac{1}{z}(Q z)^{2} K_{0}^{2}(Q z)$.

For the proton, the comparison with the experimental data [55] is shown in Fig. 8.

For the nuclear case, using the values of the parameters determined above, we obtain for the ratio $F_{L}^{A} / F_{L}^{p}$ the results in Fig. 9.

In order to evaluate the uncertainty in the extraction of the nuclear structure functions, we recall that the structure function is experimentally determined by data on the reduced cross section $\sigma_{r}$ :

$\sigma_{r}=F_{2}\left[1-f(y) \frac{F_{L}}{F_{2}}\right]$,

where 


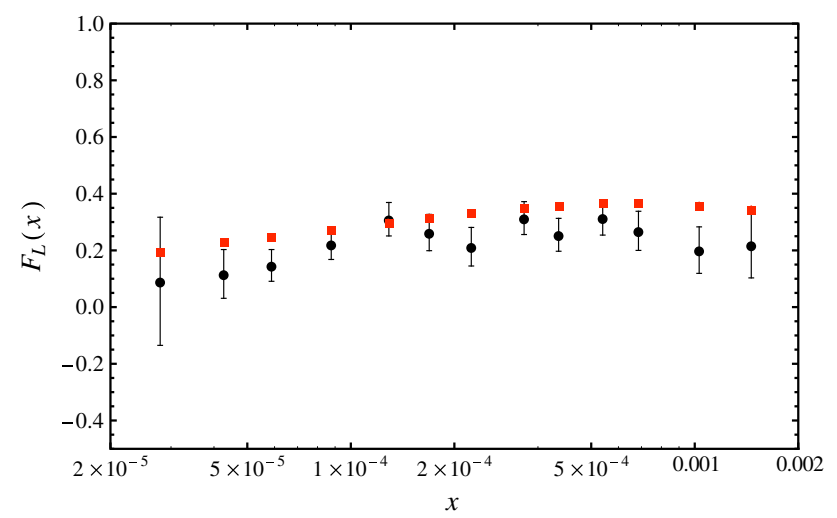

Fig. 8 Comparison with experimental data (black dots) [55] of the longitudinal structure function of the proton evaluated using the holographic formulas (red squares). The experimental $\left\langle Q^{2}\right\rangle$, from the first to the last bin in $x$, varies in the range $[1.5-45] \mathrm{GeV}^{2}$. The $\chi_{\text {d.o.f. }}^{2}$ is $\chi_{\text {d.o.f. }}^{2} \simeq 1.1$
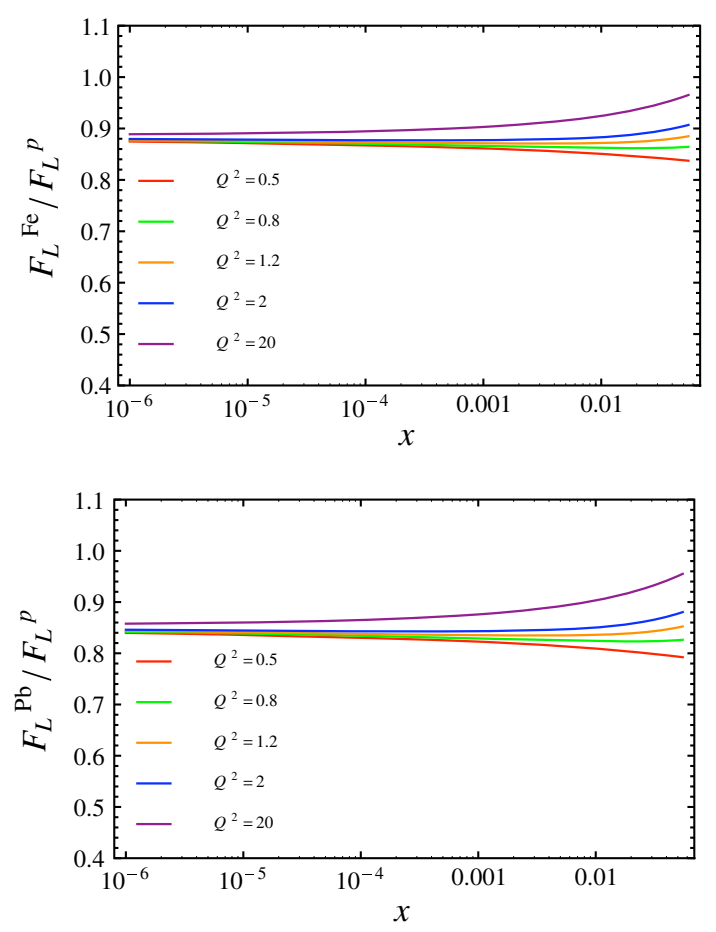

Fig. 9 Ratio between the longitudinal nuclear structure function per nucleon and for free nucleon. From top-down, the panels correspond to $\mathrm{Fe}$ and $\mathrm{Pb}$

$$
f(y)=\frac{y^{2}}{1+(1-y)^{2}} .
$$

Let us call $\hat{F}_{2}^{A}$ the structure function per nucleon; it is obtained by the relation

$\sigma_{r}=\hat{F}_{2}^{A}-f(y) F_{L}^{N}$,

i.e., using the longitudinal structure function of the free nucleon, without nuclear effects. $\hat{F}_{2}^{A}$ is an approximation of $F_{2}^{A}$ which should be determined by the relation
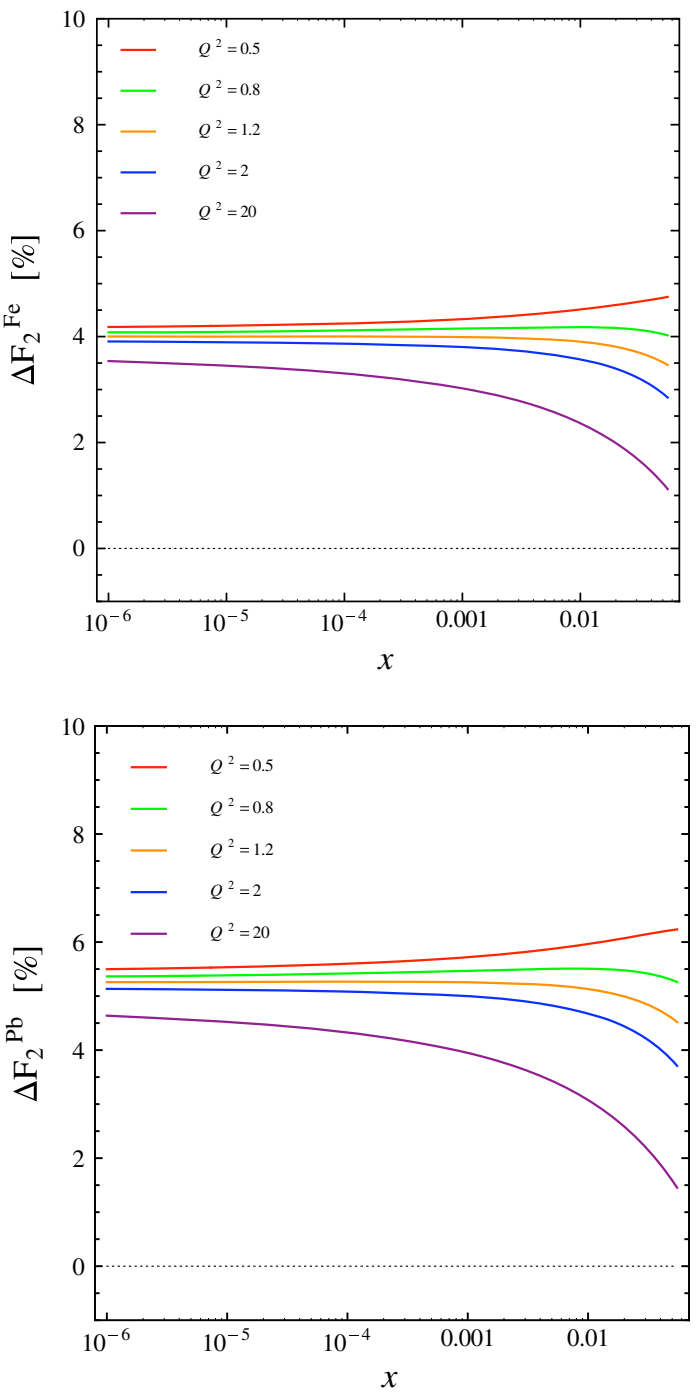

Fig. 10 Maximum uncertainty $(y=1)$ in the experimental determination of the structure function $F_{2}^{A}$ due to the absence of nuclear effects in $F_{L}$. The top panel corresponds to $\mathrm{Fe}$, the bottom one to $\mathrm{Pb}$

$\sigma_{r}=F_{2}^{A}-f(y) F_{L}^{A}$

By the expression of $F_{2}^{A}$ in Eq. (24), and using the previous equations, one can evaluate the uncertainty on $F_{2}^{A}$ :

$\Delta F_{2}^{A}=\frac{\hat{F}_{2}^{A}-F_{2}^{A}}{\hat{F}_{2}^{A}}=1-\frac{F_{2}^{A}}{F_{2}^{A}+f(y)\left(F_{L}^{N}-F_{L}^{A}\right)}$.

As shown in Fig. 10, the maximum uncertainty (corresponding to $y=1$ ) in the extraction of $F_{2}^{A}$ is of the order of a few percent also in the region of very low $x$ and $Q^{2}$.

This is consistent with the results in Ref. [56], where the longitudinal structure function in nuclear DIS at small $x$ and $Q^{2} \geq 4 \mathrm{GeV}^{2}$ is discussed in the framework of universal parton densities obtained in DGLAP analysis at next-leadingorder (NLO), with the conclusion that the uncertainty in $F_{2}^{A}$ is smaller than $10 \%$. 


\section{Conclusions and perspectives}

A description of nuclear shadowing, i.e. the distortion at low $x$ of the nuclear DIS structure functions, can be obtained by a rescaling the virtual photon momentum $Q^{2}$, and this modification naturally emerges in a holographic approach. Experimental data for electroproduction are theoretically reproduced, hence the AdS/CFT formulation captures the relevant dynamics to describe the nuclear DIS effects.

The next step of the study would be the analysis of the experimental results for DIS neutrino scattering on nuclear target, an interesting issue due to the large theoretical uncertainties in current calculations of neutrino cross section at high energy and very low $x$ [57]. Universality of nuclear effects in DIS has been recently shown [58] by the analysis of neutrino data which takes into account the different normalizations of independent experiments: the nuclear modifications are found to be the same as in electroproduction. A calculation in the holographic framework would require the solution of the equation of motion for charged currents in the bulk, to obtain an expression analogous to (7): this analysis deserves a dedicated study. For the time being, simple arguments are encouraging. Indeed, for a correct normalization procedure and to facilitate the data comparison with theory, in Ref. [58] the ratio between neutrino data on nuclear target and the theoretical proton cross section (i.e. without nuclear effects) are considered, instead of the absolute experimental cross section. The average value of this ratio, $R_{A}^{\nu}$, in the small$x$ bins, turns out to be $R_{A}^{v} \simeq 0.94 \pm 0.09$ for $x=0.015$, $R_{A}^{\nu} \simeq 1 \pm 0.08$ for $x=0.045$ and $R_{A}^{v} \simeq 1.03 \pm 0.05$ for $x=0.08$ [58]. Neglecting the contribution of the structure function $x F_{3}$ to the cross section, which should be small in the considered kinematical region, a comparison can be done between $R_{A}^{\nu}$ and the ratio $F_{2}^{F e} / F_{2}^{p}$ evaluated in the holographic approach for the corresponding average values of $x$ and $Q^{2}$. One obtains $F_{2}^{F e} / F_{2}^{p} \simeq 0.88,0.93,0.97$ for $x=0.015,0.045,0.08$, respectively, consistent with the corresponding $R_{A}^{v}$. Therefore, the approach based on the holographic method is also promising for other analyses, for instance neutrino scattering. Moreover, different ways of introducing a conformal symmetry breaking through, e.g., a background dilaton [59] represent variants worth to be investigated for the calculation of the nuclear structure functions on the basis of the ideas exploited in our study. Since the holographic method can be applied to small $Q^{2}$ values, our conclusions confirm the complementarity of the AdS/CFT inspired techniques with the perturbative calculations.

Acknowledgments Paolo Castorina acknowledges the CERN TH Unit for hospitality.

Open Access This article is distributed under the terms of the Creative Commons Attribution License which permits any use, distribution, and reproduction in any medium, provided the original author(s) and the source are credited.

Funded by $\mathrm{SCOAP}^{3}$ / License Version CC BY 4.0.

\section{References}

1. M. Arneodo, Phys. Rept. 240, 301 (1994)

2. L. Frankfurt, V. Guzey, M. Strikman, Phys. Rept. 512, 255 (2012)

3. S.V. Akulinichev et al., Phys. Lett. B 158, 485 (1985)

4. S.V. Akulinichev et al., Phys. Rev. Lett. 55, 2239 (1985)

5. F.E. Close, R.G. Roberts, G.G. Ross, Phys. Lett. B 129, 346 (1983)

6. F.E. Close, R.L. Jaffe, R.G. Roberts, G.G. Ross, Phys. Rev. D 31, 1004 (1985)

7. R.L. Jaffe, Phys. Rev. Lett. 50, 228 (1983)

8. R.L. Jaffe, F.E. Close, R.G. Roberts, G.G. Ross, Phys. Lett. B 134, 449 (1984)

9. F.E. Close, R.G. Roberts, G.G. Ross, Phys. Lett. B 168, 400 (1986)

10. R.P. Bickerstaff, G.A. Miller, Phys. Lett. B 168, 409 (1986)

11. P. Castorina, A. Donnachie, Phys. Lett. B 215, 589 (1988)

12. P. Castorina, A. Donnachie, Z. Phys, C 45, 141 (1989)

13. B. Povh, J. Hufner, Phys. Rev. Lett. 58, 1612 (1987)

14. J.M. Maldacena, Adv. Theor. Math. Phys. 2, 231 (1998)

15. J.M. Maldacena, Int. J. Theor. Phys. 38, 1113 (1999)

16. S.S. Gubser, I.R. Klebanov, A.M. Polyakov, Phys. Lett. B 428, 105 (1998)

17. E. Witten, Adv. Theor. Math. Phys. 2, 253 (1998)

18. G.F. de Teramond, S.J. Brodsky, Phys. Rev. Lett. 94, 201601 (2005)

19. J. Erlich, E. Katz, D.T. Son, M.A. Stephanov, Phys. Rev. Lett. 95, $261602(2005)$

20. O. Aharony, J. Sonnenschein, S. Yankielowicz, Annals Phys. 322, 1420 (2007)

21. J. Casalderrey-Solana, H. Liu, D. Mateos, K. Rajagopal, U.A. Wiedemann. arXiv:1101.0618 [hep-th]

22. J. Polchinski, M.J. Strassler, Phys. Rev. Lett. 88, 031601 (2002)

23. J. Polchinski, M.J. Strassler, JHEP 0305, 012 (2003)

24. C.A. Ballon Bayona, H. Boschi-Filho, N.R.F. Braga, JHEP 0803 , 064 (2008)

25. C.A. Ballon Bayona, H. Boschi-Filho, N.R.F. Braga, JHEP 0810, 088 (2008)

26. L. Cornalba, M.S. Costa, Phys. Rev. D 78, 096010 (2008)

27. L. Cornalba, M.S. Costa, J. Penedones, JHEP 1003, 133 (2010)

28. L. Cornalba, M.S. Costa, J. Penedones, Phys. Rev. Lett. 105, $072003(2010)$

29. Y. Hatta, E. Iancu, A.H. Mueller, JHEP 0801, 026 (2008)

30. Y. Hatta, E. Iancu, A.H. Mueller, JHEP 0801, 063 (2008)

31. J.L. Albacete, Y.V. Kovchegov, A. Taliotis, JHEP 0807, 074 (2008)

32. B. Pire, C. Roiesnel, L. Szymanowski, S. Wallon, Phys. Lett. B 670, 84 (2008)

33. Y.V. Kovchegov, Z. Lu, A.H. Rezaeian, Phys. Rev. D 80, 074023 (2009)

34. A.H. Mueller, A.I. Shoshi, B.-W. Xiao, Nucl. Phys. A 822, 20 (2009)

35. E. Avsar, E. Iancu, L. McLerran, D.N. Triantafyllopoulos, JHEP 0911, 105 (2009)

36. C. Marquet, B.-W. Xiao, F. Yuan, Phys. Lett. B 682, 207 (2009)

37. Y.V. Kovchegov, Phys. Rev. D 82, 054011 (2010)

38. A. Watanabe, K. Suzuki, Phys. Rev. D 86, 035011 (2012)

39. R.C. Brower, M. Djuric, I. Sarcevic, C.-I. Tan, JHEP 1011, 051 (2010)

40. L. Agozzino, P. Castorina, P. Colangelo, Phys. Rev. Lett. 112, 041601 (2014)

41. R.C. Brower, J. Polchinski, M.J. Strassler, C.-I. Tan, JHEP 0712 , 005 (2007)

42. M. Arneodo et al., New Muon. Collaboration. Nucl. Phys. B 487, 3 (1997) 
43. P. Amaudruz et al., New Muon Collaboration. Nucl. Phys. B 371, 3 (1992)

44. K.J. Eskola, H. Paukkunen, C.A. Salgado, JHEP 0904, 065 (2009)

45. K.J. Eskola, V.J. Kolhinen, H. Paukkunen, C.A. Salgado, JHEP 0705, 002 (2007)

46. A. Accardi, F. Arleo, N. Armesto, R. Baier, D.G. d'Enterria, R.J. Fries, O. Kodolova, I.P. Lokhtin et al. arXiv:hep-ph/0310274

47. P. Amaudruz et al., New Muon Collaboration. Nucl. Phys. B 441, 3 (1995)

48. M. Arneodo et al., New Muon. Collaboration. Nucl. Phys. B 441, 12 (1995)

49. M. Arneodo et al., New Muon Collaboration. Nucl. Phys. B 481, 3 (1996)

50. N.N. Nikolaev, B.G. Zakharov, Z. Phys, C 49, 607 (1991)
51. A.H. Mueller, Nucl. Phys. B 415, 373 (1994)

52. J.L. Albacete, N. Armesto, J.G. Milhano, C.A. Salgado, U.A. Wiedemann, Eur. Phys. J. C 43, 353 (2005)

53. J.L. Albacete, N. Armesto, J.G. Milhano, C.A. Salgado, U.A. Wiedemann, Phys. Rev. D 71, 014003 (2005)

54. Z. Abidin, C.E. Carlson, Phys. Rev. D 79, 115003 (2009), and in references therein

55. F.D. Aaron et al., H1 Collaboration. Eur. Phys. J. C 71, 1579 (2011)

56. N. Armesto, H. Paukkunen, C.A. Salgado, K. Tywoniuk, Phys. Lett. B 694, 38 (2010)

57. M. Kuroda, D. Schildknecht, Phys. Rev. D 88, 053007 (2013)

58. H. Paukkunen, C.A. Salgado, Phys. Rev. Lett. 110, 212301 (2013)

59. A. Karch, E. Katz, D.T. Son, M.A. Stephanov, Phys. Rev. D 74, 015005 (2006) 